\title{
TRABAJAR POR AMOR AL ARTE: \\ PRECARIEDAD LABORAL COMO FORMA DE VIOLENCIA CONTRA LOS PERIODISTAS EN MÉXICO
}

\author{
WORKING FOR THE LOVE OF ART: LABOUR PRECARIOUSNESS \\ AS A FORM OF VIOLENCE AGAINST JOURNALISTS IN MEXICO
}

\section{Rubén Arnoldo González Macías}

Benemérita Universidad Autónoma de Puebla, México

(D) https://orcid.org/0000-0002-6758-5328

\section{Dulce Alexandra Cepeda Robledo}

Universidad Autónoma de Tamaulipas, México

(D) https://orcid.org/0000-0002-3009-4330

Autor para correspondencia: Rubén Arnoldo González Macías, email: ruben.arnoldo@correo.buap.mx

\section{Resumen}

Las agresiones contra la prensa no se limitan a los asesinatos, golpizas o secuestros. Por el contrario, en países como México, los informadores enfrentan cada vez mayores riesgos, tales como amenazas, campañas de difamación o espionaje digital. A pesar de la diversidad de ataques, el tema de la precariedad laboral como una forma de violencia no ha sido estudiado en profundidad. Por lo tanto, el objetivo de este artículo es analizar la percepción que los trabajadores de medios mexicanos tienen respecto a las condiciones con las que realizan sus tareas cotidianamente. A partir de una serie de entrevistas semiestructuradas con periodistas en activo ubicados en los 23 estados más peligrosos del país, los resultados indican que las circunstancias laborales adversas obstaculizan el cumplimiento de su función, la cual es fomentar la rendición de cuentas de la clase en el poder y, como resultado, mantener informada a la sociedad. Asimismo, el origen de esta situación se encuentra en las propias organizaciones mediáticas, cuyos dueños anteponen los intereses económicos a los estrictamente periodísticos.

Palabras clave: periodismo, precariedad laboral, violencia contra la prensa, México, corrupción.

\begin{abstract}
Aggressions against the press are not limited to assassinations, beatings, or kidnappings. On the contrary, in countries such as Mexico, media staff face increasing risks like threats, smear campaigns, or digital espionage. Despite the diversity of assaults, labour precariousness as a form of violence against journalists has not been sufficiently studied. Therefore, the aim of this paper is to analyse Mexican news workers' perceptions on the
\end{abstract}


adverse conditions under which they work on a daily basis. Drawing on a set of semi-structured interviews with active journalists located in the 23 most dangerous states of the country, the findings indicate that their precarious labour conditions hinder their journalistic duties, which are fostering elite groups accountability, and citizens' right to know. In addition, the origin of this situation can be found in the organizations they work for, whose owners privilege their own economic interests over those strictly journalistic.

Keywords: journalism, labour precariousness, anti-press violence, Mexico, corruption.

Recibido: 14/04/2021

Aceptado: 02/06/2021

\section{Introducción}

La violencia en contra de la prensa se ha convertido rápidamente en un fenómeno muy complejo, en el que constantemente convergen una diversidad de actores (gobierno, crimen organizado, grupos de choque, sindicatos...) y factores (corrupción, impunidad, inconformidad social...) (Cottle et al., 2016). En ese sentido, México se ha convertido en el ejemplo paradigmático de esta situación, ya que desde hace más de una década- el país se encuentra en una espiral de violencia que ha causado la muerte de más de un centenar de periodistas (Committee to Protect Journalists [CPJ], 2021). Sin embargo, los asesinatos son solamente la punta del iceberg, puesto que existe una diversidad de agresiones que van desde amenazas y difamación, pasando por arrestos arbitrarios, hasta llegar a golpizas, secuestros y torturas (Artículo 19, 2020).

Es por lo anterior que los ataques contra los informadores se han convertido en un foco de atención académica, tanto nacional como internacional. Bajo diferentes enfoques tanto teóricos como metodológicos, los principales estudios se han concentrado en diversos ángulos, tales como la conformación de colectivos de periodistas como respuesta a los constantes ataques (De León, 2018); el impacto psicológico de cubrir temas como el crimen organizado (Flores et al., 2014; González, 2020); la erosión de las normas profesionales (González y Relly, 2016; González et al., 2021); la desconfianza social en los periodistas y sus implicaciones para las coberturas de alto riesgo (González y Reyna, 2019); o el uso de tecnologías digitales como medida de protección contra posibles agresiones durante el reporteo de temas sensibles (González y Rodelo, 2020). Asimismo, hay un creciente interés en la predicción de amenazas y agresiones, al considerar que hay ciertas regiones, temáticas y actitudes profesionales más peligrosas que otras (Hughes y Márquez-Ramírez, 2018; Salazar, 2019).

No obstante, hay un tema que ha permanecido ausente en la literatura académica, el cual es el de la precariedad laboral como una forma 
de violencia en contra de los periodistas. A pesar de que es una preocupación constante para ellos (Beltrán, 2019; Valdez, 2016), este aspecto ha sido escasamente analizado. Una posible explicación que será retomada más adelante apunta a que es bien sabido que los salarios en los medios son bajos, por lo que quien se quiera dedicar a esta profesión debe aceptar trabajar así. Es decir, muchos reporteros, fotógrafos, camarógrafos, editores o conductores han naturalizado esta situación y, por ende, la dan por sentada.

Si bien el tema salarial ha sido mencionado constantemente en no pocos estudios (ver por ejemplo Espino Sánchez, 2016; González, 2013; Maldonado, 2018; Márquez, 2014; Reyna, 2018), su abordaje ha sido más tangencial que central. Lo mismo puede decirse de las condiciones laborales adversas como una forma de violencia, lo cual apenas comienza a delinearse (Del Palacio, 2018; CepedaRobledo, 2018; Del Palacio el al., 2020), sin llegar todavía a un estudio más centrado y profundo.

Es por lo anterior, que el objetivo de este artículo es analizar la precariedad laboral como una manera de atacar a los periodistas y, como resultado, discutir las implicaciones que esta situación tiene para la realización de su trabajo. Para lograrlo, esta investigación parte de una serie de entrevistas semiestructuradas con informadores mexicanos que laboran en los 23 estados con mayor incidencia de violencia en el país (Artículo 19, 2019).

Los hallazgos indican que los bajos salarios y la ausencia de todo tipo de prestaciones sociales (servicios de salud, pago de vacaciones, aguinaldo...) vulneran el bienestar de los trabajadores de medios, obligándolos al multiempleo, recibir sobornos o vender publicidad, todo en detrimento de su labor profesional. De acuerdo con los entrevistados, esta situación es fomentada por los propios dueños de los medios, quienes anteponen los intereses económicos por encima de los periodísticos.

Para desarrollar de mejor manera estas ideas, el documento se organiza de la siguiente forma: La primera sección presenta una revisión de la literatura sobre los orígenes de la precariedad laboral, el caso de México, y la violencia contra la prensa en este país. En la segunda se detalla la metodología usada en este estudio, seguida por el análisis de los resultados. Finalmente, el artículo cierra con la discusión de los hallazgos y sus implicaciones para entender las condiciones adversas de trabajo en las que los periodistas mexicanos desarrollan su trabajo.

\section{Revisión de la literatura}

\section{Precariedad laboral}

Desde las primeras investigaciones de Engels (1845) en el que se aplicó la metodología marxista para conocer el modo de vida y trabajo de los obreros ingleses a finales del siglo XIX, ya se visualizaba una estrecha relación entre el modo de producción, la actividad del trabajo social y el modo de vida. Del mismo modo, Marx y Engels (1932) explicaron los fundamentos de las sociedades basadas en el trabajo social, en el que este aspecto y el económico se 
complementan de forma que se configuran en la división del trabajo y las formas de propiedad. Estas primeras investigaciones de los sociólogos surgieron debido a la observación de las distintas clases de trabajo entre obreros y burgueses de la época, que manifestaban una marcada diferencia en sus estilos de vida y trabajo.

En el Manifiesto Comunista (1848), ambos autores explicaban que, debido a la expansión de la clase burguesa, al ser despojados los trabajadores de los medios de subsistencia agraria, sólo les quedaba vender su fuerza de trabajo; pero bajo condiciones laborales inseguras y con contratos que la mayoría de las veces eran verbales y sin garantías. Dichas condiciones han mejorado con el paso de los años, pero no en todas las regiones del mundo se observan las mismas circunstancias.

El origen etimológico del término "precariado" proviene del latín "precari" que significa "pedir, rezar, rogar; por ello, inseguridad, dependencia del favor de otro, inestabilidad, exposición al peligro; con una incierta permanencia" (Breman, 2014, p. 148). Standing (2011) define al precariado como los trabajadores de medio tiempo, con contratos temporales o mano de obra subcontratada, pero que no forman parte de la clase trabajadora o proletariado, que, en cambio, pueden tener acceso a sindicatos y seguridades laborales.

Los trabajadores de los medios de comunicación y/o periodistas se encuentran entre estas dos definiciones: existen tanto los que tienen sus contratos a largo plazo con horarios y prestaciones definidas, como los que aspiran a tenerlas a través de un largo periodo de inestabilidad laboral, méritos, bajos salarios y, particularmente, agresiones. En ambos casos la jornada no está establecida: existe hora de entrada, pero no de salida.

La explotación del trabajador de los medios de comunicación sucede para mantener el statu quo de los dueños de los medios de producción de noticias. Es decir, hay una tendencia hacia "(1) reducir costos, (2) limitar o reducir el número de trabajadores permanentes, (3) maximizar la flexibilidad de sus empresas, y/o (4) trasladar sus riesgos hacia los trabajadores" (Kallenberg, 2012, p. 142). En principio, los tres primeros objetivos obedecen a la lógica del mercado, pero no proveer a los periodistas de lo mínimo necesario es atentar contra ellos, especialmente en las condiciones de extrema violencia que se viven en distintas ciudades, tal y como se discutirá en el contexto del periodismo mexicano.

\section{El caso de México}

Dentro de la amplitud de los estudios de periodismo en México, la precariedad laboral de los informadores ha sido un tema poco explorado. Si bien frecuentemente se hace alusión a él, son más bien escasos sus análisis específicos $\mathrm{y}$, menos recurrente aún, su asociación con el panorama general de la violencia cotidiana que enfrentan los informadores en este país. Por lo tanto, en esta sección se presenta una mirada general de esta situación, que servirá de contexto para explicar los resultados de esta investigación.

Las limitadas condiciones de trabajo que enfrentan aquellos que laboran en la producción de 
noticias han sido asumidas como algo inherente al periodismo mexicano (Merchant Ley, 2019; Reyna, 2018). Aunque sin ser propiamente el hilo conductor, algunos estudios recientes se han referido a este aspecto como una de las múltiples aristas que limitan el desarrollo de los medios. Por ejemplo, la baja rentabilidad que aqueja a las organizaciones periodísticas -dependientes casi por completo de los convenios de publicidad gubernamental- fomenta la precariedad laboral y ésta, a su vez, el clientelismo y la corrupción (Maldonado, 2018; Márquez, 2014; Martínez et al., 2009; Salazar, 2018). En otras palabras, los bajos salarios y escasas prestaciones que reciben muchos de los informadores le abren la puerta al chayote $^{1}$, el cual representa un complemento de su sueldo, pero compromete su independencia editorial (González, 2013; Maldonado, 2018; Márquez, 2014).

$\mathrm{Si}$ bien las condiciones poco favorables de empleo son recurrentes en los medios de todo el territorio nacional, los periodistas ubicados en el interior de los estados -fuera de las capitales o cabeceras municipales- tienden a ser los más precarios (De León, 2018; De León et al., 2018; Del Palacio, 2018; Espino Sánchez, 2016; Merchant Ley, 2019). Es precisamente en esas regiones donde la transición democrática del país no ha representado cambios significativos en la relación prensa-poder, lo cual es sólo uno entre otros muchos rezagos, tanto del sistema mediático como del político (Espino Sánchez, 2016, González, 2018; González y

\footnotetext{
${ }^{1}$ En el argot periodístico mexicano, el término chayote indica el dinero que reporteros, fotógrafos, editores y hasta conductores de noticieros reciben -principalmente
}

Echeverría, 2018; Hughes y Márquez-Ramírez, 2018).

Aunado a lo anterior, la desventajosa situación contractual de los trabajadores de los medios es un factor que -tarde o temprano-impacta en la calidad de su trabajo. Dicho de otro modo, mientras se realice en condiciones adversas, el periodismo profesional es insostenible a largo plazo (Merchant Ley, 2019; Reyna, 2017). Esto es especialmente evidente en coberturas de temas potencialmente peligrosos, en las que las carencias laborales inhiben la decisión de afrontar los riesgos asociados a estos (Cepeda-Robledo, 2018; Medel, 2010). Por ende, la precariedad laboral -asumida como una baja valoración de su trabajo por parte de los empleadores- detona las intenciones de renuncia de los periodistas y, en ciertos casos, hasta de abandonar el periodismo (Reyna, 2017).

Como se mencionó antes, considerar la precariedad laboral como un tipo de violencia ejercida en contra de los informadores es un ángulo que no ha sido propiamente analizado como un problema específico de investigación. Sin embargo, estudios recientes han sugerido que las condiciones desfavorables que enfrenta la mayoría de los reporteros, fotógrafos/camarógrafos y editores, entre otros, representan en efecto una agresión (CepedaRobledo, 2018; Del Palacio, 2018). Si bien no pueden equipararse con los secuestros, torturas o asesinatos, los bajos salarios y prestaciones sociales también son un factor que vulnera el quehacer

de alguna instancia de gobierno o partido político-a cambio de una cobertura favorable hacia determinados personajes. 
periodístico (Blanco-Herrero et al., 2020; CepedaRobledo, 2018; Del Palacio, 2018, Del Palacio et al., 2020).

Es precisamente este último punto el que los mismos periodistas han empezado a hacer público (Beltrán, 2019; Valdez, 2016). Desde diferentes regiones del país han surgido voces del propio gremio que denuncian las condiciones poco favorables en las que tienen que realizar su ya de por sí riesgoso trabajo. Partiendo de su condición de afectados directos, están visibilizando las implicaciones que para su labor tienen los salarios bajos, inestabilidad laboral, prestaciones limitadas o inexistentes, despidos injustificados y debilitamiento de sindicatos, por mencionar algunos aspectos. Esta situación, aseguran, fomenta un periodismo de baja calidad.

\section{Violencia contra la prensa en México}

El siglo XXI ha sido particularmente peligroso para el periodismo en gran parte del mundo. De acuerdo con el Comité para la Protección a Periodistas (2021), 1,648 periodistas han sido asesinados a nivel mundial. Durante ese mismo periodo, 120 reporteros fueron víctimas de ataques mortales en México, lo que coloca al país como uno de los lugares más peligrosos del planeta para la prensa (CPJ, 2021). En ese sentido, el objetivo de esta sección es presentar una mirada general de las condiciones de riesgo a las que permanentemente se enfrentan los periodistas mexicanos, para lo cual se discutirán cuatro temas clave: diversidad de agresiones, agresores y víctimas; dispersión geográfica de los ataques; colusión entre autoridades y agresores; e impunidad.

La violencia contra los periodistas mexicanos es un fenómeno multifacético, ya que no se limita exclusivamente a asesinatos. Por el contrario, hay una diversidad de agresiones que los trabajadores de los medios constantemente tienen que enfrentar (Artículo 19, 2020. A pesar de que los ataques físicos (golpizas, tortura, secuestros...) son las agresiones más evidentes y extremas, también hay una serie de ataques menos visibles tales como amenazas, acoso personal y digital, campañas de desprestigio e intervención de dispositivos electrónicos, entre otros (Artículo 19, 2020 González y Rodelo, 2020).

En ese sentido, el amplio abanico de agresiones ha generado un igualmente diverso grupo de agresores y víctimas. Por un lado, diferentes actores han sido consistentemente señalados de presuntamente cometer actos violentos en contra de informadores. Entre los más comúnmente acusados son autoridades de gobierno, políticos, miembros del crimen organizado, caciques, ciertos empresarios, y hasta manifestantes (Artículo 19, 2020 Del Palacio, 2018; Washington Office on Latin America [WOLA] y Peace Brigades International [PBI], 2016). Por otro lado, a pesar de los riesgos inherentes a la cobertura de la llamada Guerra contra las Drogas y/o corrupción gubernamental (Hughes y MárquezRamírez, 2018; Salazar, 2019), dichos reporteros ya no son las únicas víctimas de ataques. Por el contrario, hay un creciente número de periodistas que cubren otras fuentes de información (local, deporte y hasta espectáculos) que también han sido afectado 
(Artículo 19, 2020 Del Palacio, 2018; González y Reyna, 2019).

A pesar de que los asesinatos de periodistas están más o menos concentrados en estados específicos como Tamaulipas, Chihuahua, Sinaloa o Veracruz (Del Palacio, 2018; Hughes et al, 2017; Relly y González de Bustamante, 2014), otro tipo de agresiones son comunes en todo el territorio nacional (Artículo 19, 2020 González y Reyna, 2019; González y Rodelo, 2020). Por lo tanto, hay una percepción generalizada de riesgo latente entre los periodistas mexicanos, aunque no necesariamente trabajen en una zona particularmente peligrosa (González y Reyna, 2019).

Esta situación es frecuentemente promovida por la colusión entre ciertas autoridades de gobierno y los agresores (Artículo 19, 2020 De León y González, 2020; Del Palacio, 2018; Hughes et al, 2017; Relly y González de Bustamante, 2014; WOLA y PBI, 2016). De tal suerte, este fenómeno presenta dos caras: en primer lugar, en no pocas ocasiones algunos servidores públicos son cooptados por grupos de poder (crimen organizado, cacicazgos, élites políticas...), cuyos miembros son señalados como agresores (Relly y González de Bustamante, 2014; Hughes et al, 2017; Del Palacio, 2018; De León y González, 2020). En segundo lugar, hay algunos estados como Veracruz en donde los principales sospechosos de atacar a la prensa son las propias autoridades del gobierno estatal (Artículo 19, 2020; Del Palacio, 2018; WOLA y PBI, 2016).

Por lo tanto, se puede sugerir que la diversidad de ataques contra periodistas, su distribución geográfica, y la colusión entre ciertos actores de gobierno y criminales, es resultado de la impunidad generalizada en el sistema político mexicano. Es decir, al igual que la mayoría de los crímenes en el país, casi todos los ataques contra la prensa quedan impunes (Artículo 19, 2020 De León y González, 2020; Del Palacio, 2018; Hughes et al, 2017; WOLA y PBI, 2016). La razón de ello es que casi nueve de cada diez de dichas acusaciones no se resuelven, debido a que muchas ni siquiera son investigadas propiamente (De León y González, 2020).

\section{Metodología}

El presente estudio es de corte cualitativo y se organiza a partir de una serie de entrevistas semiestructuradas con informadores mexicanos, realizadas entre febrero de 2017 y agosto de 2018. La muestra comprendida por 93 informantes -62 hombres y 31 mujeres- fue seleccionada a partir de dos criterios: en primer lugar, debían ser periodistas en activo, ya fueran empleados de alguna redacción o freelancers. En segundo lugar, se requería que, en el momento de la entrevista, cubrieran -o hayan cubierto consistentemente- hard news, especialmente temas de delincuencia organizada o corrupción. No fue criterio de exclusión su rol (por ejemplo, reportero, editor, fotógrafo, camarógrafo, conductor), ni el tipo de medio para el que trabajaran (periódico, revista, radio, televisión, o portal de noticias). 
La selección de entrevistados siguió la lógica del muestreo no probabilístico por bola de nieve, puesto que fueron los mismos periodistas quienes iban recomendado a otros colegas a participar en el estudio. Dentro de la conformación de la muestra final, se condujeron entrevistas con reporteros locales y corresponsales estatales de medios nacionales (ubicados en la Ciudad de México), tales como Proceso, La Jornada, Reforma, El Universal, Televisa, TV Azteca, e Imagen Televisión. Asimismo, participaron periodistas de reconocidos medios locales y regionales como El Informador, El Siglo, A.M., El Diario de Juárez, Zeta y Río Doce, por nombrar algunos. También se entrevistó a informadores de portales independientes de noticias locales, además de personal de agencias de noticias nacionales e internacionales (por ejemplo, Notimex, EFE y $A F P$ ).
El cuestionario aplicado incluyó ocho preguntas base, mismas que se organizaron en tres grandes temas: contexto general de la violencia contra periodistas en México, impacto de las agresiones, y seguridad digital (Tabla 1). Cabe señalar que la precariedad laboral no fue incluida explícitamente en ninguna pregunta. Sin embargo, dicho aspecto surgió de forma espontánea en las respuestas obtenidas del primer bloque del instrumento, específicamente cuando se les interrogó sobre “¿Por qué se ataca a la prensa en México?”, "¿Quiénes son los principales agresores de periodistas?” y “¿Cuáles son las principales agresiones que sufren los periodistas?". En ese sentido, y dada la frecuencia con la que se hizo alusión al tema por parte de 24 de los 93 entrevistados (justo la cuarta parte), se decidió presentar este reporte específico al respecto.

\section{Tabla 1}

Cuestionario base aplicado a los participantes del estudio

\begin{tabular}{cl}
\hline Tema & \multicolumn{1}{c}{ Preguntas } \\
\hline \multirow{3}{*}{ Contexto } & ¿Por qué se ataca a la prensa en México? \\
& ¿Quiénes son los principales agresores de periodistas? \\
& ¿Qué opina de los mecanismos institucionales de protección a periodistas? \\
\hline & ¿De qué manera impacta al periodista, como individuo, las agresiones en su \\
& contra y/o de sus compañeros? \\
& ¿De qué manera impacta al medio de comunicación que se agreda a uno de sus \\
periodistas? & ¿De qué manera impacta a la sociedad las agresiones contra la prensa? \\
\hline \multirow{2}{*}{ Seguridad digital } & En términos de seguridad personal y/o digital del periodista, ¿qué opina del uso \\
& de las tecnologías digitales de comunicación, tales como teléfonos inteligentes \\
& y redes sociales? \\
\hline
\end{tabular}


Con la finalidad de que la investigación tuviera un alcance nacional, las entrevistas se distribuyeron geográficamente siguiendo, por un lado, la zonificación del Consejo Nacional para la Seguridad Pública y, por el otro, los reportes presentados por Artículo 19 en el 2016 y 2017 (Artículo 19, 2019), en los que se señala el número de agresiones contra periodistas y el lugar donde ocurrieron. En ese sentido, la distribución de la muestra se enfocó en los 23 estados de la República más peligrosos reportados por dicha ONG, y divididas en las regiones propuestas por el antes mencionado programa del gobierno federal (Tabla 2).

\section{Tabla 2}

Distribución geográfica de la muestra

\begin{tabular}{cl}
\hline Zona & Muestra por estado seleccionado \\
\hline Noroeste & Baja California: 9; Chihuahua: 6; Sinaloa: 6; Sonora: 5 \\
\hline Noreste & Coahuila: 3; Nuevo León: 4; San Luis Potosí: 4; Tamaulipas: 4 \\
\hline Occidente & Aguascalientes: 1; Guanajuato: 2; Jalisco: 6; Michoacán: 7; Zacatecas: 3 \\
\hline Centro & Ciudad de México: 4; Estado de México: 2; Guerrero: 2; Morelos: 1; Puebla: 8 \\
\hline Sureste & Chiapas: 3; Oaxaca: 3; Quintana Roo: 4; Tabasco: 1; Veracruz: 5 \\
\hline
\end{tabular}

Finalmente, debido a las consideraciones éticas con Resultados las que se realizó esta investigación, la identidad de los informantes se mantendrá en el anonimato. Es decir, puesto que el carácter sensible de las respuestas podría comprometer a los entrevistados, se determinó hacer referencia a ellos mediante la letra $E$ (Entrevistado) y un número (por ejemplo, E12). Asimismo, para contextualizar y dimensionar sus respuestas, solamente se indicará su rol dentro del medio y la región donde labora.

Del análisis de las respuestas emergieron tres variables asociadas a la precariedad laboral como una forma de violencia contra los periodistas mexicanos: intereses económicos versus intereses periodísticos, condiciones laborales precarias y corrupción ${ }^{2}$. De tal suerte, la presentación de los resultados seguirá ese orden.

\footnotetext{
${ }^{2}$ Las variables aquí reportadas son resultado de un análisis inductivo de las respuestas, el cual siguió las pautas de la Teoría Fundada. Es decir, se codificaron los datos, se establecieron patrones de relación entre ellos, y - como resultado- se desarrollaron dichas variables. Sin embargo,
}

tal y como marca la antes mencionada teoría, estas últimas no fueron clasificaciones preconcebidas. Por el contrario, emergieron conforme se desarrolló el trabajo de campo, puesto que fue la misma evidencia empírica la que las determinó (Charmaz, 2006; Charmaz y Belgrave, 2015). 


\section{Intereses económicos versus intereses periodísticos}

Al cuestionar sobre los principales agresores contra los periodistas, algunos entrevistados pudieron identificar claramente a los dueños de los medios de comunicación para los que trabajan como los autores de los agravios. Tal y como se aprecia a continuación:

Mira, la respuesta fácil y el lugar común es que [los principales agresores] son los funcionarios del gobierno, pero la situación es mucho más compleja. Me parece que los principales enemigos de los periodistas son precisamente los dueños de los medios. Esto se da porque ellos tienen intereses políticos y comerciales que son más importantes que sus propios empleados (E51, conductor de noticiero y editor de un periódico local, Región Noreste).

Complementa esta explicación otro entrevistado al indicar lo siguiente:

Las empresas de comunicación que ganan millones y millones en contratos de publicidad oficial y, además, que nos censuran, nos marcan una línea, nos denigran, y nos tienen como una

3 Manuel Guerrero (2016) clasifica a la "censura ambiental" en la que el periodista colabora repitiendo los boletines de prensa sin diversificar o corroborar sus fuentes, sigue su línea editorial y depende de la publicidad oficial. El periodismo "responsivo" sería el que responde a diversos temas de lectores exigentes, usa las nuevas herramienta para ir ganando dinero ellos y dejarnos a nosotros con sueldos miserables. (E65, editor de una revista regional, Región Noroeste).

De acuerdo con Manuel Guerrero (2016), hay tres tipos de ejercicio periodístico en México: la censura ambiental, el periodismo responsivo y el periodismo acosado $^{3}$. Es este último el que se vive en la mayor parte de la República Mexicana, principalmente en ciudades pequeñas de provincia con pocos anunciantes, gran dependencia de la publicidad oficial, sin lectores comprometidos o exigentes. Lejos de los grandes corporativos multimediáticos millonarios que existen en la capital del país, los reporteros locales de municipios más pequeños, los que sólo son conocidos por sus pocos lectores, vecinos, familiares y fuentes cercanas, se encuentran en la total vulnerabilidad, en la precariedad y fuertemente acosados para entrar a dinámicas corruptas que terminan por cobrar la vida del propio periodista; tanto si publica la verdad como si no lo hace. Un periodista del norte del país explica esta situación:

$$
\begin{aligned}
& \text { Imagínate que algún gobierno tiene una } \\
& \text { pauta importante en un medio de mayor } \\
& \text { circulación o que tiene mayor audiencia, y si } \\
& \text { hay un reportero incómodo pues entonces el }
\end{aligned}
$$

tecnologías y se ubica en las grandes ciudades. Finalmente, el periodismo "acosado" tiene lugar en pequeñas y medianas ciudades, hace señalamientos o denuncias a las instituciones, pero en cambio es hostigado por el crimen organizado y grupos políticos. 
mandatario habla con el dueño o el director general y le dice 'tú me estás pidiendo tanto, y éste viene con sus preguntas incómodas, $y$ luego le dejas que hasta tenga una columna'. Entonces el dueño llega con el reportero y le dice 'oye de aquí comemos todos y espérame tantito'. Esas son las formas. La pauta es una forma de control por parte del gobierno. Hay que decirlo: los medios deberían generar un modelo de negocio que fuera independiente y que no sólo viviera de las pautas gubernamentales (E82, reportero de un periódico local, Región Noroeste).

\section{Condiciones laborales precarias}

Continuando con otra de las consecuencias de un bajo salario también se encuentran las condiciones laborales precarias. No es la norma que los dueños de los medios se comprometan a proporcionar las herramientas, capacitación, equipo o transporte necesario para realizar las coberturas de noticias. Por el contario, estos gastos deben ser financiados por los mismos reporteros con su propio sueldo, tal como se aprecia en la siguiente respuesta:

Los dueños de los medios también contribuyen a esta situación [violencia contra periodistas] al no proveer las herramientas necesarias para hacer tu trabajo. Por ejemplo, si no tienes auto, debes usar el transporte público. Otras veces hasta tienes que usar tu propio equipo, y ellos no te dan un seguro en caso de robo o daño
(E26, fotógrafa de un periódico local, Región Occidente).

Nueve periodistas entrevistados en diversas partes del país coinciden en calificar las condiciones laborales precarias, salarios bajos y falta de seguridad social por parte de los dueños de los medios de comunicación como una agresión frecuente y sistemática contra la prensa. "Esa es la realidad del periodismo en México: trabajamos por amor al arte. Nada más" (E53, reportero de un periódico local, región Noreste). Esta situación no siempre mejora con la antigüedad del periodista:

La violencia más peligrosa es cuando los reporteros son abandonados por los medios para los que trabajan. Los dueños ni les pagan un salario digno ni les otorgan seguridad social. Conozco varios periodistas ya viejos que viven en la miseria (E61, reportero de un portal de noticias locales, Región Sureste).

Estas condiciones laborales no son ignoradas por los grupos de poder, que precisamente buscan "comprar" los favores y silencios a cambio del chayote. Hay algunos políticos quienes incluso agreden psicológica y públicamente a los reporteros al humillarlos frente a sus compañeros:

Algunos alcaldes lo hacen en sus discursos al denostar públicamente al periodista, diciendo que es un muerto de hambre, $y$ muchos otros comentarios despectivos. Por decirte un ejemplo: 'pobrecitos, los 
entiendo, pues ganan tres pesos ¿verdad? Pues con cualquier cosa que les paguen está bien'. (E70, reportera de un periódico local, Región Noroeste).

Otro aspecto asociado a la precariedad es la inseguridad laboral, la cual es más sutil, sistemática y se puede confundir frecuentemente con acoso, debido a que permanentemente se amenaza al reportero con perder el empleo y/o ser vetado, no solamente en su empresa si no en la región.

Cuando hablamos de violencia contra la prensa mucha gente piensa en agresiones físicas. Sin embargo, aquí [Región Noreste] las agresiones son más sutiles pero constantes, sistemáticas y eficientes. Una agresión es que te corran de tu trabajo, porque afectaste los intereses de alguien más. Después de eso, ya nadie te vuelve a contratar otra vez. Por eso, vivimos constantemente al filo de la navaja. Podemos perder nuestro trabajo en cualquier momento, y perder tu trabajo aqui significa perderlo en todo el estado (E51, conductor de noticiero y editor de un periódico local, Región Noreste).

Desde otra región de la República, otro reportero coincide y explica que "el gobierno presiona a los dueños de medios para que despidan a los reporteros incómodos, los cuales difícilmente vuelven a ser contratados por otra organización" (E62, corresponsal de una televisora nacional, Región Noroeste). En ese sentido, la penalización por exponer las malas acciones del gobierno no sólo es la precariedad, sino también inseguridad laboral. Así lo señala $E 78$ (reportero de un periódico local Región Noroeste): "lo veo no solamente desde el aspecto de la violencia física, de la criminalidad, sino también de la violencia laboral, que en algún momento puedes llegar a perder el trabajo por algún comentario que hagas".

A esta situación se agrega la ya latente dificultad de realizar las coberturas cuando se trata de informar sobre situaciones de peligro derivadas del crimen organizado:

Frecuentemente, el editor sentado tras su escritorio te ordena que cubras un choque o un tiroteo, pero ni él ni tú saben si la víctima pertenece a algún cártel; porque, si ese es el caso, seguido pasa que algún sicario regresa para asegurarse que la persona esté muerta. El editor no sabe, o ni le importan los riesgos que corremos a diario. Él sólo te dice 'se tiene que hacer, y si no lo haces tú, atrás de ti hay 20 chavos que quieren tu trabajo por la mitad de tu sueldo' (E50, fotógrafo de un portal de noticias locales, Región Noreste).

\section{Corrupción}

Como resultado de buscar el interés económico por encima del periodístico, y las condiciones laborales adversas que ello implica, se desprenden dos situaciones que favorecen la corrupción. En primer lugar, el periodista busca una remuneración extra vendiendo su silencio o espacios publicitarios, según 
convenga a los políticos en el poder. Es decir, pidiendo o aceptando chayote, o incluso el narcochayote. Esta situación se resume en la lógica de "salario mínimo y manos libres", que según E51 (conductor de noticiero y editor de un periódico local, Región Noreste), consiste en pagarle al reportero lo menos posible, pero no obstaculizar ninguna otra medida que decida aplicar para obtener dinero extra. Al realizar esta práctica, se compromete la calidad o pertinencia de la información que se publica. Las siguientes respuestas ilustran este punto:

Este es un estado muy pobre [Región Sureste] y muchos colegas enfrentan condiciones laborales muy adversas. A muchos de ellos rara vez les pagan. Esta situación fomenta la corrupción. Este tipo de violencia indirecta es económica, y te obliga a actuar en contra de los valores periodísticos... La violencia económica tiene un impacto evidente en el periodismo de calidad (E54, reportera de un portal de noticias locales, Región Sureste).

Sin exagerar, la mayoría de los periodistas ganamos entre tres y cuatro mil pesos. Incluso me atrevería a decir al mes. No nos alcanza y las empresas periodísticas tienen mucho que ver. No estoy diciendo y no estoy justificando que hagamos un trabajo para el chayote, en pro del chayote, pero sí te estoy diciendo que uno de los grandes problemas es que si existe el chayote es porque las empresas periodísticas no están haciendo bien su chamba (E65, editor de una revista regional, Región Noroeste).

$\mathrm{Si}$, por el contrario, el periodista no compromete su integridad y la información ante los grupos de poder (o sea, se resiste a ser comprado), los mismos políticos se encargan de agredirlo para hacerlo desistir: "Ellos [los miembros del gobierno y el crimen organizado] buscan amedrentar a la prensa cuando no la pueden comprar" (E69, reportero de un periódico local, Región Noroeste). Otro participante de este estudio lo expresa del siguiente modo:

Los políticos usan su poder económico para controlar a los medios. Esto pasa muy frecuentemente a través de los convenios de publicidad oficial. Los chayotes también cumplen esa función, debido a las injustas condiciones laborales en las que muchos periodistas tienen que trabajar (E61, reportero de un portal de noticias locales, Región Sureste).

Tal como lo explica otra entrevistada, frecuentemente la corrupción generada por la precariedad laboral implica también un riesgo para la vida de los periodistas:

No sólo hay un factor que permite el final trágico para la vida de un periodista. Hay muchos factores, incluyendo la corrupción entre los mismos reporteros. No se puede decir que los periodistas son víctimas porque sí. Por el contrario, esas víctimas estaban 
dentro de un contexto en el que ellas estaban involucradas. La corrupción se da porque los salarios son muy bajos (E17, corresponsal de un periódico nacional, Región Sureste).

\section{Discusión y conclusiones}

En páginas anteriores se señalaba que la violencia contra periodistas es un fenómeno multifacético en México, porque intervienen diferentes tipos de víctimas, agresores y agresiones. También se hacía hincapié en el hecho de que la precariedad laboral no había sido discutida sistemáticamente -al menos en el ámbito académico- como una forma de violencia en contra de quienes trabajan en los medios de comunicación produciendo noticias. En ese sentido, el presente estudio contribuye con la literatura especializada al incluir dicho tema en el debate científico, y fomentar su posterior análisis.

Por lo que respecta a los datos aquí presentados, se destaca que -a pesar de no haber sido incluida como una pregunta específica- la precariedad laboral emergió espontáneamente en las respuestas de la cuarta parte de los entrevistados, lo que indica el nivel de importancia que representa este aspecto para ellos. Asimismo, el hecho de que no todos los participantes lo hayan expresado abiertamente no necesariamente implica que no se vean afectados por ésta. Por el contrario, lo anterior puede sugerir que es algo asumido como natural y que se da por sentado. No obstante, este punto requiere un estudio que específicamente pueda explorar esta hipótesis.

De acuerdo con los informantes, la precariedad laboral implica trabajar a cambio de salarios bajos, sin prestaciones ni seguridad social. Esto se da frecuentemente porque la relación con las organizaciones sigue el esquema de pago por honorarios, puesto que no hay contratos laborales entre ambas partes. Asimismo, en no pocas ocasiones los periodistas trabajan con su propio equipo. Esta situación obliga a los reporteros a tener múltiples empleos, ya sea en otros medios o en áreas ajenas al campo. Además, hay un constante estado de inestabilidad laboral, debido a la alta rotación de personal, despidos frecuentes, y hasta vetos.

Los participantes de esta investigación afirman que el origen de este fenómeno se encuentra precisamente en los propios medios de comunicación. Es decir, señalan un sabotaje de los mismos dueños de las organizaciones para las que trabajan, quienes ponderan los intereses económicos por encima de los periodísticos. Desde este punto de vista, esto genera que la autonomía para realizar coberturas de temas sensibles (como la corrupción de ciertas autoridades gubernamentales o actividades ilícitas de otros actores políticos y sociales) se vea significativamente reducida, porque los propietarios de los medios están más interesados en maximizar sus ganancias, aunque esto vaya en detrimento de la función social del periodismo, que es fomentar la transparencia y la rendición de cuentas.

En ese sentido, los intereses mediáticos no pasan desapercibidos en el trabajo de los periodistas, que deben seguir la línea editorial que les es fijada 
desde la dirección del medio. Se trata de contratos arreglados entre las altas esferas gubernamentales y empresariales, en las cuales la calidad de la noticia, la línea editorial o el mismo silencio es la moneda de cambio; incluso más importante que el propio espacio publicitario. Estas prácticas periodísticas no sólo atentan contra la percepción del gremio en la sociedad, o contra la transmisión de noticias oportunas, veraces, y relevantes; si no que además deteriora el contrapeso de debe realizar el medio de comunicación ante los grupos de poder elegidos democráticamente.

Ante este panorama, los periodistas mexicanos tienen tres opciones para revertir esta situación: la primera es tener varios empleos y/o vender publicidad, la segunda es aceptar sobornos y ser parte de la corrupción, y la tercera es abandonar la profesión. Los tres escenarios suponen un impacto negativo tanto para el periodismo como para la sociedad. Con respecto a lo primero, una autonomía profesional tan acotada fomenta una práctica periodística pasiva (cobertura de eventos oficiales y dependencia en boletines), autocensura, difusión de propaganda y existencia de zonas de silencio (lugares $\mathrm{y}$ temas que no reciben atención mediática). Al cumplir un rol más de vocero que de periodista profesional, no se desarrolla la función de exigir rendición de cuentas a los grupos en el poder y, por ende, no se promueve el derecho de los ciudadanos de estar informados sobre temas de interés público.

Estas condiciones adversas a las que se enfrentan los periodistas en México, situados principalmente en las pequeñas y medianas ciudades, además de vulnerar su bienestar, merma la calidad de la información que requiere la sociedad. De este modo, se mantiene una dinámica forzada por el miedo y las amenazas, deteriorando los pocos contrapesos que el periodismo ejerce sobre los grupos de poder. Ante esta situación, la producción de noticias se convierte en una mercancía que se vende y con la que los beneficiados terminan siendo los empresarios de medios. Su estrategia es tan efectiva que quienes son señalados en la opinión pública como chayoteros o vendidos son los propios periodistas, no así los dueños de las organizaciones para las que trabajan (González y Reyna, 2019; González y Rodelo, 2020).

Bajo estas circunstancias, el concepto de periodismo acosado (Guerrero, 2016) discutido anteriormente puede explicar mejor la dependencia que existe entre la situación de vulnerabilidad y precariedad salarial del periodista y los contratos de los dueños de los medios de comunicación con los grupos de poder. Entre más compromisos editoriales tienen los empresarios, menos libertad de expresión tiene el medio de comunicación para ser contrapeso de los grupos políticos. Como resultado, se ve mermada la calidad de la información que ofrece el medio, ya que utiliza a sus periodistas como meros recolectores de datos, sin importar los riesgos a los que se enfrentan, el desgaste o costo de sus equipos, ni las actualizaciones que deben pagar para enfrentar los retos del periodismo digital.

Cabe señalar que la precariedad laboral de los informadores no es un tema exclusivo de México, ya que en diferentes regiones del Sur Global esta situación es cada vez más frecuente (ver, por ejemplo, Blanco et al., 2020; Matthews y 
Onyemaobi, 2020). Por lo tanto, su análisis también puede abordarse desde diferentes perspectivas. Por ejemplo, este fenómeno se ha presentado consistentemente en el resto de las llamadas industrias culturales, de las que los medios de comunicación forman parte (Mastrini y Becerra, 2007; Sánchez, 2007). Es decir, debido a la naturaleza glamorosa de este tipo de organizaciones, los jóvenes estudiantes y/o recién egresados de las universidades gustosamente ofrecen su talento a cambio de poco y nada (de Peuter et al., 2015). Por lo tanto, este esquema de trabajo no retribuido fomenta un sistema de explotación no regulado, ya que, con la promesa de ofrecerles experiencia, grandes y pequeñas empresas se sirven del talento joven para generar ganancias a muy bajos costos (de Peuter et al., 2015; Hesmondhalgh, 2015; Ursell, 2000).

Asimismo, las experiencias recogidas en este estudio pueden ser explicadas desde el enfoque de la Economía Política de la Comunicación (Mosco, 2009; Sparrow, 1999; Wald, 1987), ya que enfatizan los conceptos de "control y supervivencia" de los medios (Mosco, 2009, p. 3). Es decir, para asegurar la operación de sus empresas y la generación de ganancias, los dueños de las organizaciones periodísticas estrechan relaciones con las élites políticas y económicas, aunque eso afecte su quehacer periodístico. Por tanto, en un mercado mediático tan saturado como el mexicano, un adecuado desempeño financiero -no informativo- es prioritario (Espino Sánchez, 2019; Gómez, 2020; Mosco, 2009; Sparrow, 1999; Wald, 1987). De ahí que, como lo manifiestan los mismos periodistas, las condiciones laborales de sus empleados no son la prioridad de la mayoría de los empresarios de medios.

Tal y como se ha reconocido a lo largo del texto, éste es un primer acercamiento más o menos formal al estudio de la precariedad laboral como una forma de violencia en contra de los periodistas en México; un tema que emergió de forma espontánea en una investigación más amplia sobre las constantes agresiones, tanto internas como externas, que obligan a los informadores mexicanos trabajar "por amor al arte”. Por tanto, se requieren nuevos estudios que analicen en específico este aspecto, lo cual podría resolverse mediante una encuesta con una muestra más amplia y robusta, que indague con mayor profundidad los temas aquí discutidos. Además, con el fin de contribuir al desarrollo teórico de este fenómeno, las futuras investigaciones sobre el tema podrían enmarcarse en alguno de los dos enfoques arriba mencionados.

\section{Referencias bibliográficas}

Artículo 19. (2019). Looking back, looking forward: Mexico. Global Expression Report 2018/19: The State of Freedom of Expression around the World. https://bit.ly/3xmEHg8

Artículo 19. (2020). Primer semestre de 2020: crecen exponencialmente las agresiones contra la prensa y continúan los asesinatos. https://bit.ly/3xCYs31 
Beltrán, J. A. (2019). Por primera vez en Sinaloa, un Congreso llama a los medios a pagarle IMSS a periodistas. Noroeste. https://bit.ly/3vuuP2s

Blanco-Herrero, D., Oller Alonso, M., y Arcila Calderón, C. (2020). Las condiciones laborales de los periodistas iberoamericanos. Diferencias temporales y geográficas en Brasil, México, Chile, España y Portugal. Comunicación y Sociedad, 17, 1-39. https://doi.org/10.32870/cys.v2020.7636

Breman, J. (2014). Un concepto espurio. New Left Review, 84(enero-febrero 2014), 143-152. https://bit.ly/3gpPmRI

Cepeda-Robledo, D. A. (2018). Violentómetro periodístico del sur de Tamaulipas, Axon, 2, 196-204. https://bit.ly/3wuWjGJ

Charmaz, K. (2006). Constructing Grounded Theory. A practical guide through qualitative analysis. Sage Publications.

Charmaz, K., y Belgrave, L. (2015). Grounded Theory. En G. Ritzer (Ed.), The Blackwell Encyclopedia of Sociology (pp. 1-6). John Wiley and Sons.

Committee to Protect Journalists [CPJ] (2021). 120 Journalists and Media Workers Killed in Mexico between 2000 and 2021/Motive Confirmed or Unconfirmed. https://bit.ly/35nBmBx

Cottle, S., Sambrook, R., y Mosdell, N. (2016). Reporting dangerously. Journalist killings, intimidation and security. Palgrave McMillan

De León, S. (2018). Profesionalización autogestiva de los periodistas mexicanos organizados. Global Media Journal México, 15(28), 78-99. https://doi.org/10.29105/gmjmx15.28-6

De León, S., Bravo, A., y Duarte, E. M. (2018). Entre abrazos y golpes... Estrategias subpolíticas de periodistas mexicanos frente al riesgo. Sur le journalisme, About journalism, Sobre jornalismo, 7(1), 114-129. https://bit.ly/3vzXWBO

De León, S., y González, R. A. (2020). Reportear en el desamparo: Análisis de las medidas de protección a periodistas en México desde el contexto local. Revista de Comunicación, 19(2), 87-109. https://doi.org/10.26441/RC19.2-2020-A5

de Peuter, G., Cohen, N. S., y Brophy, E. (2015). Interrogating Internships: Unpaid Work, Creative Industries, and Higher Education. Introduction. Triple C, 13(2), 329-335. https://bit.ly/3xrFIUd

Del Palacio, C. (2018). Callar o morir en Veracruz. Violencia y medios de comunicación en el sexenio de Javier Duarte (2010-2016). Juan Pablos Editor.

Del Palacio, C., Gómez, G., y Salazar G. (2020). Condiciones laborales y de seguridad de los periodistas en contextos de violencia en Iberoamérica. Comunicación y Sociedad, 17, 1-11. https://doi.org/10.32870/cys.v2020.7805

Engels, F. (1845). La situación de la clase obrera en Inglaterra. Marxist.org. https://bit.ly/3pXWuYt

Global Media Journal México, 18(34), 209-228, enero - junio 2021. 
Espino Sánchez, G. (2016). Periodistas precarios en el interior de la República Mexicana. Atrapados entre las fuerzas del mercado y las presiones de los gobiernos estatales. Revista Mexicana de Ciencias Políticas y Sociales, 61(228), 1-30. https://bit.ly/3gFNvXR

Espino Sánchez, G. (2019). El nuevo sistema de medios en el México del siglo XXI. Fontamara.

Flores, R., Reyes, V., y Reidl, L. (2014). El impacto psicológico de la guerra contra el narcotráfico en periodistas $\begin{array}{lllll}\text { mexicanos. Revista Colombiana de Psicología, 23(1), } & \text { 177-193. }\end{array}$ https://www.doi.org/10.15446/rcp.v23n1.37640

González, C., y Relly, J. (2016). Professionalism Under Threat of Violence: Journalism, Reflexivity, and the Potential for Collective Professional Autonomy in Northern Mexico. Journalism Studies, 17(6), 684-702. https://www.doi.org/10.1080/1461670X.2015.1006903

Gonzalez, R. A. (2013). Economically-driven partisanship-Official advertising and political coverage in Mexico: The case of Morelia. Journalism and Mass Communication, 3(1), 14-33. https://bit.ly/2U0EQaC

González, R. A. (2018). Silence or alignment. Organized crime and government as primary definers of news in Mexico. (OBS*) Observatorio Journal, 12(4), 125-139. https://doi.org/10.15847/obsOBS12420181127

González, R. A. (2020). Mexican Journalism Under Siege. The Impact of Anti-press Violence on Reporters, Newsrooms, and Society. Journalism Practice, 15(3), 308-328. https://doi.org/10.1080/17512786.2020.1729225

González, R. A., y Echeverría, M. (2018). A medio camino: El sistema mediático mexicano y su irregular proceso de modernización. Revista Mexicana de Opinión Pública, 13(24), 35-51. https://doi.org/10.22201/fcpys.24484911e.2018.24.60437

González, R. A., y Reyna, V. H. (2019). “They don’t trust us; they don’t care if we're attacked”: trust and risk perception in Mexican journalism. Communication \& Society, 32(1), 147-160. http://dx.doi.org/10.15581/003.32.1.147-160

González, R. A., y Rodelo, F. (2020). Double-edged knife: practices and perceptions of technology and digital security among Mexican journalists in violent contexts. Tapuya: Latin American Science, Technology and Society, 3(1), 22-42. https://doi.org/10.1080/25729861.2020.1746502

González, R. A., Díaz-Cerveró, E., y Barredo-Ibáñez, D. (2021). Reportear bajo amenaza: violencia, profesionalización y modernización irregular del sistema mediático mexicano. Palabra Clave, 23(4), 127. https://doi.org/10.5294/pacla.2021.24.1.1

Gómez, R. (2020). El rol del Estado en el Sistema de Medios Mexicano 2013-2018. Punto de partida para una agenda de investigación. Comunicación y Sociedad, 17, 1-28. https://doi.org/10.32870/cys.v2020.7565

Guerrero, M. A. (2016). Democracia y medios en México: el papel del periodismo. Instituto Nacional Electoral. https://bit.ly/3gCOt7c

Global Media Journal México, 18(34), 209-228, enero - junio 2021. 
Hesmondhalgh, D. (2015). Exploitation and Media Labour. En R. Maxwell (Ed.), The Routledge Companion to Labor and Media (pp. 1-20). Routledge.

Hughes, S., y Márquez-Ramírez, M. (2018). Local-Level Authoritarianism, Democratic Normative Aspirations, and Antipress Harassment: Predictors of Threats to Journalists in Mexico. The International Journal of Press/Politics, 23(4), 539-560. https://doi.org/10.1177\%2F1940161218786041

Hughes, S., Mellado, C., Arroyave, J., Benitez, J. L., de Beer, A., Garcés, M., Lang, K., Márquez-Ramírez, M. (2017). Expanding influences research to insecure democracies. Journalism Studies, 18(5), 645-665. https://doi.org/10.1080/1461670X.2016.1266278

Kallenberg, A. (2012). Globalización y trabajo precario: Desafíos para la sociedad y la sociología. En A. Cárdenas, F. Linky, y J. Stillerman (Eds), ¿Qué significa el trabajo hoy? Cambios y continuidades en una sociedad global (pp. 41-53). Catalonia.

Maldonado, P. (2018). El salario de los periodistas, el ancla a su participación en las redes de clientelismo mediático. Global Media Journal México, 15(28), 1-16. https://doi.org/10.29105/gmjmx15.28-1

Márquez, M. (2014). Professionalism and journalism ethics in post-authoritarian Mexico: perceptions of news for cash, gifts, and perks. En W. Wyatt (Ed.), The ethics of journalism: individual, institutional and cultural influences (pp. 55-63). I.B. Tauris, Reuters Institute for the Study of Journalism.

Martínez, S., Matus, J., y Villar, H. A. (2009). La condición laboral del periodista en Chiapas. Revista Latina de Comunicación Social, 12(64), 444-460. https://bit.ly/2SGclyC

Marx, C., y Engels, F. (1848). Manifiesto Comunista. Akal.

Marx, C., y Engels, F. (1932). La ideología alemana. Ediciones Pueblos Unidos. https://bit.ly/3xpgS7A

Mastrini, G., y Becerra, M. (2007). Globalización, mercado e industrias culturales: ¿Resistencia o simulacro?. Diálogos de la Comunicación, 75, 1-7. https://bit.ly/3vAsWRY

Matthews, J., y Onyemaobi, K. (2020). Precarious Professionalism: Journalism and the Fragility of Professional Practice in the Global South. Journalism Studies, 1-17. https://doi.org/10.1080/1461670X.2020.1797524

Medel, M. (2010). Periodismo en tiempos de amenazas, censura y violencia. Centro Knight para el Periodismo en las Américas.

Merchant Ley, D. D. (2019). "Cuando reporteas te expones": la precarización de la labor periodística en Baja California. Carta Económica Regional, 31(123), 99-119. https://doi.org/10.32870/cer.v0i123.7633

Mosco, V. (2009). The political economy of communication. Sage.

Relly, J., y González de Bustamante, C. (2014). Silencing Mexico: A Study of Influences on Journalists in the Northern States. The International Journal of Press/Politics, 19(1), 108-131. https://doi.org/10.1177\%2F1940161213509285

Reyna García, V. H. (2017). Estudios culturales y laborales del periodismo: una revisión bibliográfica. Balajú. Revista de Cultura y Comunicación, 6(4), 3-21. https://bit.ly/2S3bhVs

Global Media Journal México, 18(34), 209-228, enero - junio 2021. 
Reyna, V. H. (2018). La puerta giratoria entre el periodismo y la comunicación política en Sonora. En M. Echeverría, y R. A. González (Coords.), Comunicación política y crisis de la democracia. Nuevos entornos $y$ desafios (pp 105-128). Tirant Lo Blanch, BUAP.

Salazar, G. (2019). Strategic allies and the survival of critical media under repressive conditions: An empirical analysis of local Mexican press. The International Journal of Press/Politics, 24(3), 341-362. https://doi.org/10.1177\%2F1940161219843200

Salazar, M. G. (2018). ¿Cuarto poder? Mercados, audiencias y contenidos en la prensa estatal mexicana. Política y Gobierno, 25(1), 125-152. https://bit.ly/3gtB0zC

Sánchez, E. (2007). Industrias culturales, diversidad y pluralismo en América Latina. Global Media Journal México, 4(7), 31-45. https://bit.ly/35tgZTG

Sparrow, B. H. (1999). Uncertain guardians: The news media as a political institution. The Johns Hopkins University Press.

Standing, G. (2011). The precariat: The new dangerous class. Bloomsbury Academic.

Ursell, G. (2000). Television production: issues of exploitation, commodification and subjectivity in UK television

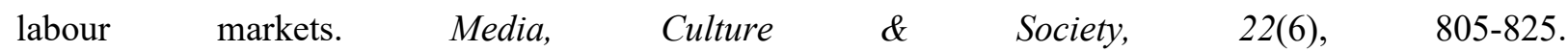
https://doi.org/10.1177\%2F016344300022006006

Valdez, J. (2016). Narcoperiodismo. La prensa en medio del crimen y la denuncia. Aguilar.

Wald, R. C. (1987). The news marketplace: A ride on the truth machine. Gannett Center Journal, 1(1), 7-20.

Washington Office on Latin America [WOLA], y Peace Brigades International [PBI]. (2016). Mexico's mechanism to protect Human Rights defenders and journalists. Progress and continued challenges. WOLA PBI. 